\title{
Writing a Successful Grant Application ${ }^{1}$
}

\author{
JOHN E. NIEDERHUBER, M.D. \\ Department of Microbiology/Immunology and Surgery, University of Michigan \\ Medical Center, Ann Arbor, Michigan 48109
}

Submitted for publication December 20, 1984

Every investigator has experienced the arduous task of preparing a research grant for submission to a funding agency. The amount of investigator time involved in this process has increased during recent years with a decline in available research funds and an increase in the number of applicants. Indeed, the competition for available research dollars has never been more intense, requiring that the application be carefully and thoroughly prepared.

Several previous studies have examined the shortcomings of applications which appeared to be the cause of a low priority score or disapproval [1,2]. In general, the characteristics which most frequently resulted in a poor application were (a) a weak or trivial hypothesis, (b) an inadequately detailed description of the research plan, and (c) a proposal which lacked focus. The purpose of this communication is to provide the young investigator, new to the academic faculty, with some guidelines in writing a successful grant application.

\section{SOURCES OF FUNDING}

One of the most frequently asked questions is "Where should I apply?" To answer this involves some work on the part of investigator. Every research university has several sources of what is commonly referred to as "seed money" and it is important to determine what funds are available and how to apply for them. Examples are the Biomedical Research Support Grant, moneys awarded to the institution

\footnotetext{
' Presented at Society of Surgical Oncology Symposium on "Grantsmanship: How to Write and Review Grant Applications," New York, May 13, 1984.
}

by NIH based on the amount of federal funding to the Medical school and the American Cancer Society Institutional Research Support Grants. In addition, new faculty can seek support from appropriate state and national foundations such as the Kidney Foundation, the Heart Foundation, and the American Cancer Society.

Federal support is available from several sources including the National Institutes of Health, The National Science Foundation, The Department of Defense, and the Veterans Administration. The latter requires a signifcant appointment fraction in your institutions Veterans Administration Hospital.

\section{THE NIH GRANT REVIEW PROCESS}

In order to understand why research applications are disapproved or rated poorly, it is helpful to know the process used for review [2]. Investigator initiated research project grants, termed R01 applications by NIH, are received by the Division of Research Grants, and assigned to an appropriate review panel of scientists known as a Study Section. The application is also assigned to an appropriate NIH Institute or Division. The Study Section is assisted by an Institute based Executive Secretary who assigns each application to a primary and secondary reviewer. At the scheduled meetings of the Study Section, the assigned reviewers lead a discussion of the scientific merit of the proposal. A vote is conducted to approve or disapprove the application, and if approved, members of the Study's Section vote a priority score. A summary statement of the critique, referred to as the 
"pink sheet," is prepared by the Executive Secretary and is available to the applicant.

The application and the results of the initial review are then presented to the Institute's or Division's advisory council responsible for program review. Council determines how well the proposed research would advance the institute's mission and what funds are available to support external applications. Council relies heavily on the Study Section review and the priority score for the application in determining which applications will be funded. Thus, the most critical determinants of success reside in the initial review by the primary reviewers and the panel of scientists serving as members of the Study Section.

\section{WRITING THE APPLICATION}

While every application format is unique to the source, a discussion of the principles used when applying to the NIH should provide the basis for essentially all grant writing.

Section 1. Section 1 of the NIH-R01 application is designed to assemble the essential data base concerning the investigator, the institution from which the investigator is making the application, the environment and resources available to the investigator for the conduct of the proposed project, and the requested budget. Various aspects of this information are used by grants administration and, of course, by the primary reviewer and Study Section that evaluates the grant. A detailed instruction booklet accompanies each application and is very useful in the completion of Section 1. In addition, your institution has individuals in their Grants Administration Office who are knowledgeable about completing grant applications; it is important to identify these individuals and make use of their experience. It may also be helpful to obtain an $\mathrm{NIH}$ proposal from a senior investigator with an established "track record"- - preferably an individual who is actively serving on a study section at NIH-and to use that grant as a model application.

The budget portion of Section 1 deserves specific comment. For example, it is frequently tempting to "pad" the budget anticipating budget cuts during review. Such temptations, however, should be avoided and the budget written honestly and fairly. An unrealistic budget reflects poorly on the applicant and can unnecessarily influence the score voted by the Study Section. The budget for subsequent years should include annual adjustment of salaries and fringe benefits and annual budget increases for inflation.

In addition to developing a realistic budget, the applicant must provide justification for each of the budget items, such as personnel, supplies, travel, equipment, etc. This is a very important part of the application which is often taken much too lightly. These budget justifications must be thoroughly, carefully, and skillfully described in order to ensure maximum support for the project.

The Biographical Sketches of the investigator and any co-investigators should present the best possible image of the applicant. For example, it is important to demonstrate adequate training and to show how this training relates to the proposed research. The bibliography should also support the qualifications of the applicant to do the proposed research and should show a common thread for the applicants research efforts.

The application must also document other grant support currently under the control of the Principal Investigator (PI) or Co-investigators. This documentation should have sufficient detail to allow the reviewer quick and easy comprehension. An outline format is most helpful with a sentence or two which describe the objective of each listed grant. These descriptions should demonstrate that while there is no overlap between grants, the overall theme of the investigators research program is cohesive.

While reviewers of grants are usually familiar with the various institutions and departments supporting the application, it is important to describe in detail the laboratory resources available for the proposed project and to characterize the environment surrounding the investigator's laboratory. The application can be strengthened with the mention of wellknown, established investigators who work in 
the same general vicinity as the applicant's laboratory and the applicant should indicate where cross-collaborations may develop during the project.

Section 2. Research Plan-As was noted, one of the most decisive parts of the entire application is the subsection which deals with the design of the proposed experiments and the methodologies that will be used to meet the objectives described. This portion of the application, where most points are gained or lost, is extremely critical to the final rating of the proposed project. Despite its importance, the Research Plan is most often the last portion of the application written. In so doing, the applicant uses a disproportionately small amount of time writing the experimental design and methods compared to the hours spent developing and writing the Significance, Specific Aims, and detailing the Preliminary Studies or the progress of the research proposed.

Indeed, perhaps the most important advice that can be given is to begin working on the experimental design and methods first and to budget at least two-thirds of the time allotted to writing the application to devcloping this part of the application. This section needs to be detailed enough to permit the reviewers to judge the applicant's knowledge of potential problems that may arise in carrying out the proposed experiments and should provide convincing evidence that the applicant can develop the necessary flexibility and alternative procedures to meet each of these challenges as they occur. The methodologies need to be thoroughly, albeit concisely, described.

It is important that this section of the grant be well focused and that there be a logical schedule for the completion of the project. The outline describing these plans needs to be extremely precise. It may be helpful to introduce this section with an algorithm or flow chart that summarizes the experimental design in response to the stated objectives (see Fig. 1). Remember, the busy reviewer needs every assistance you can provide in comprehending what you propose to do and how you will reach your objectives. Above all, be sure that the research planned is focused on the objectives.
Of course, any successful application relies on the investigator having a significant idea or hypothesis. This hypothesis must not only be interesting and exciting but entirely plausible. Once the hypothesis/objective of the proposed research has been well defined, it is necessary to devise a series of specific aims which will provide the answers to the questions raised by the hypothesis. These specific aims are developed as clear statements of the questions to be asked and the experiments that will specifically address each specific aim. In addition, the investigator should indicate how the specific aims of the proposed project will fit into the overall, long-range objectives of the laboratory.

An excellent format for assisting the reviewer in understanding the hypothesis, the objectives, and the specific aims that will address these objectives is an outline (Fig. 2). Each specific aim is listed separately and in the logical sequence they will be addressed during the performance of the project. Under each aim, a list of proposed experiments designed to address the specific aim is provided in an outline format.

The Significance subsection is next within Section 2. Here the applicant is limited to three pages and must demonstrate a thorough knowledge of the subject upon which the project is based. This section should be used to demonstrate an understanding of the relevant and current questions concerning the proposal. The investigator must relate the project's specific aims to the critical questions being asked by the scientists working in this area of research. From reading the Significance it should be clear that a successful completion of the proposed experiment(s) will make a meaningful contribution to the knowledge base of the proposed subject.

The Preliminary Studies subsection follows the Significance and must not exceed eight pages of text. A successful application should describe enough completed preliminary work to convince the reviewer that the project has an excellent chance of being carried to a successful completion. The Preliminary Studies subsection is also the applicant's opportunity 


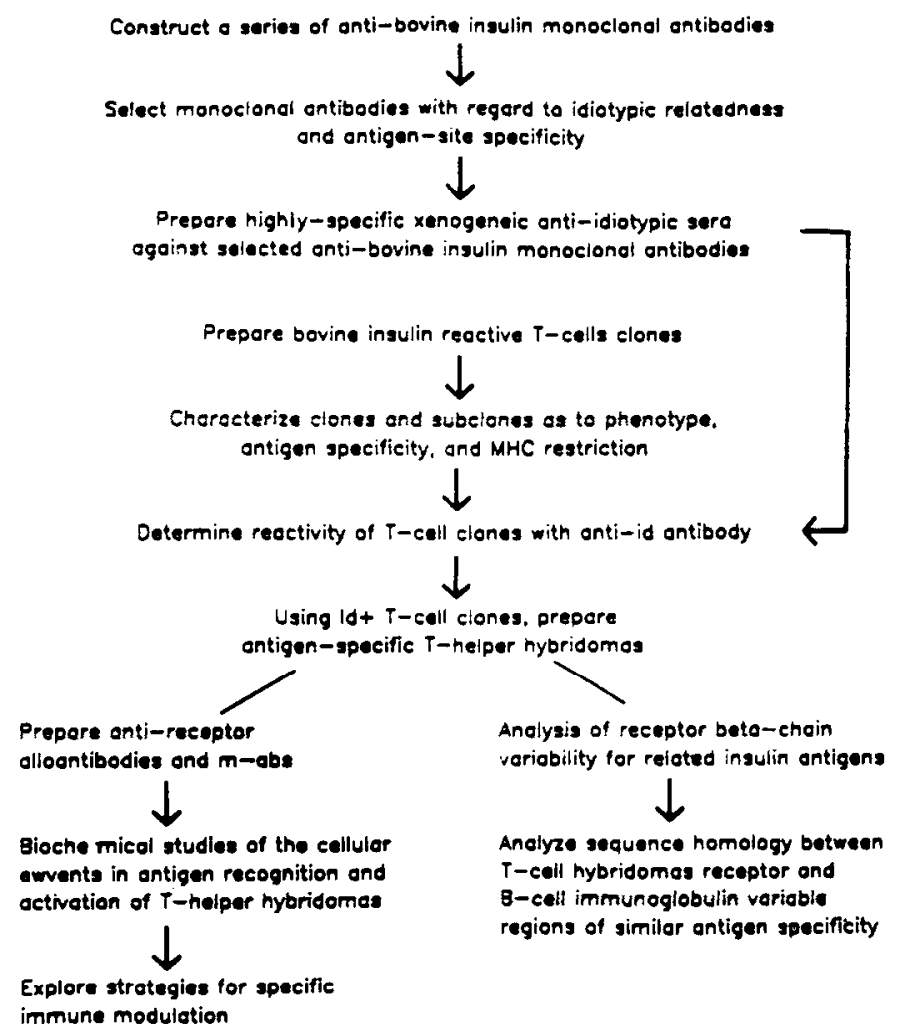

FIG. 1. This figure is taken from an original grant application and illustrates a format that can be used to present the specific aims of the proposal. Each specific aim is followed by a series of statements indicating the experimental approach to each aim.

to convince the reviewer that he or she has the skills and experience needed to competently perform the proposed experiments. For the reviewer, it is useful to have this subsection introduced by an outline which can be used to key the specific areas of the text of the Preliminary Studies.

It is extremely helpful to the reviewer to present the data obtained in Preliminary Studies in the form of tables and graphs. The graphs and tables should be of the quality used for publication. They can be reduced in size by using photocopy techniques and actually inserted in appropriate places in the text to make the job of review as easy as possible (Fig. 3). This technique is preferred to that of attaching the figures and tables as appendices requiring the reviewer to go back and forth between text and the appendix. Each table and figure should be well described with appropriate legends. Also note that the page space used by the inserted figures and tables does not count against the eight pages permitted for text.

As discussed before, the section on Experimental Design and Methods is the most important subsection of the whole application. As mentioned previously, it is the evaluation of this part of the presentation that will count the most toward the voting of the priority score. Introduce this section by a diagram showing the research planned; this helps the reviewer focus on the flow of the planned experiments and clarifies how the various parts of the Research Plan relate to each other (Fig. 1). The flow diagram also demonstrates how 


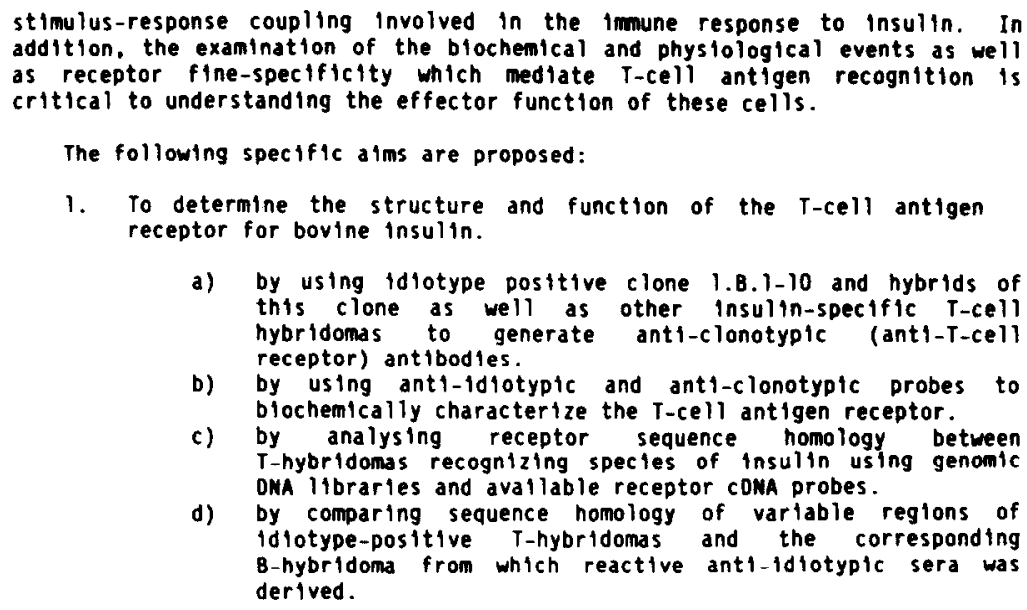

2. To determine the blochemical and physfological events assoclated with $T$-cell antigen recognition.

a) by determining changes in phosphorylated proteins in antigen-activated and resting antigen-reactive $T$ cell clones using double-labeling.

b) by using anti-idiotypic and anti-clonotypic antibodies to modulate endogenous phosphorylation.

FIG. 2. The incorporation of the figures and graphs in the text greatly aids the reviewer. This illustrates a figure reduced in size. Figures and tables should be of publication quality.

each of the steps will address the questions proposed in the Spccific Aims. I find this visual format to be quite helpful during the pressures of conducting a review of a lengthy application.

In the text of the methods, it is important to be detailed without being diffuse. Lack of focus is one of the most frequent comments in the critique of grant applications. It may be helpful to devote the first portion of the Experimental Design and Methods subsection to a detailed description of routine methods to be used and keep this description separate from the research plan. As noted earlier, the applicant should demonstrate a thorough understanding of the potential problems that might arise in the conduct of the proposed experiments and how alternative methods could be used to correct or resolve these problems.

If the application is a clinical study, statistical support is essential and the experimental plan must document methods for patient accrual and numbers of patients required to meet specific statistical objectives. It is also impor- tant to demonstrate sound procedures for data collcction, management, and analysis.

The Methods section should also contain a logical schedule for completion of the project within the time frame of requested funding. Above all, the applicant must instill in the reviewer every confidence that he or she possesses the technical skills and research background necessary to perform the experiments as proposed.

\section{PROGRAM PROJECT GRANTS}

Young investigators frequently ask questions concerning the differences between R01 individual investigator grant and P01 program project grants. These questions arise as new faculty are being introduced to various options of funding support for their research programs. Often they are called upon or have the opportunity to participate as a co-investigator with a specific project as part of a larger program grant. The P01 program project applications are obviously submitted by senior in- 


\section{Reactivity of $T$-Cell clone with Ant1-Idlotypic Antibody}

In order to investigate the effect of anti-1dlotyple antibody raised against BI monoclonal antibodies in modulating the antigen-specific proliferative response of $T$ cells, a number of $T$-cell clones were examined. Serfal dilutions of anti-ldlotypic antibody were added to $r$ cells and antigen I hour before addition of the appropriate irradiated syngenelc spleen cells. In all, 46 BI specific clones, 35 PI specific clones and 17 dual-speciftc clones, all derived from $B A L B / C$ mice were analyzed. In addition, to confirm antigen and MHC spectficity of the antisera, bovine insulin specific clones derived from C57BL/6 mice and OVA-specific clones prepared from BALB/C were assayed.

One BI-speciflc clane, designated 1.B.1-10 demonstrated a 77 . reduction in $B I$ specific proliferation when treated with anti-idiotyplc antisera ratsed agalnst hybridoma 5.288 at the lowest dilution used (Figure 3 ). No other

O-5.280 INMBITION OF INSULIN-SPECIFIC PROLIFERATION

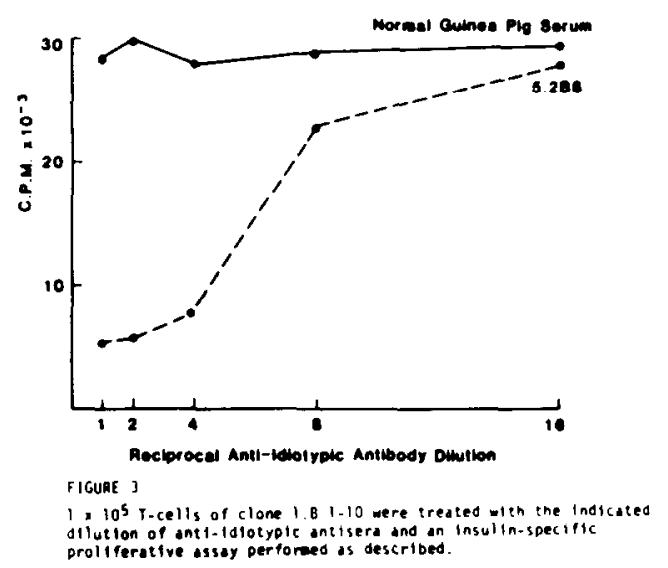

insulin-specific T-cell clone tested showed this pattern of reactivity. The
inhibition seen with this antisera or any of the other anti-idiotypic antisera
was not due to cytotoxiclty, because in these and all experiments described
below. the viability of the T cells during the assay was unaffected as judged
by trypan blue dye exclusion. In these experiments, normal guinea pig serum
was not found to be suppressive or cytotoxic.

FIG. 3. This figure illustrates a flow diagram constructed to facilitate review and to clearly present the plans for accomplishing the objectives.

vestigators with established research track records. The program project is developed around a unified and defined research goal. It is composed of strong individual projects which complement each other and which by being part of the overall program are greatly strengthened in their own right. The success of a program project application depends on the ability to demonstrate the advantage of pursuing the individual research projects as a total program rather than individual R01s. That is, the whole must be greater than the individual parts. The program is designed to enhance collaboration among the individual investigators, thus, it is important that the program not be diffuse but be well focused and of an appropriate size (appropriate number of projects).

Each of the investigators functioning as the director of one of the core projects of the program must be an established, well-recognized, funded researcher. It is essential that the application demonstrate how these project leaders relate to each other. This can best be accomplished by demonstrating a track record of joint publications and of involvement as co-investigators on $\mathrm{R} 01$ grants.

The principal investigator of the program project should be a senior scientist with recognized leadership capabilities. He or she 
should have demonstrated administrative competence and it is important that it be apparent that the individual have enough effort allotted to be an effective principal investigator for the program project. It should be noted that the quality and focus of the individual projects in the program will reflect on the principal investigator's leadership and judgment. These two qualities are important to the reviewers in judging the competence of the principal investigator and the likely success of the program project.

Adequate space and equipment must be available for the performance of the program. It is important to demonstrate that the individual project laboratories have proximity to each other which will enhance collaboration. It is also important to show that the research environment in which this program resides is such that it will enhance the interaction of the program investigators with other scientists at the institution. Finally, the research commitment of the school to this and other similar programs should be well documented.

Each P01 application requests support for several core and administrative facilities. It is critical to demonstrate how these core facilities relate to each of the described projects. That is, how important is the core to each project? The importance of the core is best demonstrated by the frequency of its use by the individual investigators. At the time of the site visit, it is helpful to have log books documenting actual investigator use available to the review panel. It is important to demonstrate in the application and at the site visit the impact of the core facilities on the efficiency of conducting the research and the quality of the research. If possible show how the core facility will result in decreased costs of the individual projects.

In documenting the administrative arrangements for the program project, it is important to indicate how the home institution or school will support the project and how the PI functions in the decision making process of the institution. Demonstrating that the PI has access to other institutional fiscal resources for the program will strengthen the application.

A site visit will be conducted by NIH with the purpose of measuring those imponderable aspects of the proposed program project-details which cannot be adequately described in a written application. This is a very critical aspect of the review process and a great deal of attention and time should be invested in seeing that the site visit team is impressed with the proposed program. It is essential that the investigators be well organized and practiced prior to the conduct of the site visit. The Executive Secretary can provide important assistance in preparing for the site visit but too often the applicant fails to use this assistance. The investigators must make it easy for the reviewers to get all the critical facts related to the project and how the objectives of the research proposed will be satisfied through the Research Plan.

The program must be composed of projects that relate well to each other and presented as a focused theme. It must be demonstrated that the accomplishments of the overall program will be greater than if these projects were performed as individual R01s. That is, synergy and collaboration are the themes of the program project. The likely success of the application depends on the fact that excellent R01 projects are focused on a common goal and that by the use of core facilities one can enhance the productivity of the individual investigator. A positive review by the site visit team depends on the ability to adequately and convincingly communicate these features. In this regard, a practice site visit using external consultants is essential.

\section{SUMMARY}

In summary, the research application must focus on a solid objective and be supported by a clear and concise plan for its accomplishment. For the new faculty member applying the first time for outside research funding, it is important to seek help from experienced and more senior colleagues, especially those 
who are actively serving on NIH Study Sections. It is a good idea to remember that the individuals reviewing applications are some of our busiest scientists. The job of grant review is clearly an added assignment that they willingly donate their time to and one needs to consider this when writing a grant application. Anything that can be done to make the reviewer's job easier can only help the application. Brevity, clarity, and organization are essential to a good application. As has been repeatedly mentioned, one of the major application weaknesses sited is a lack of focus, especially in the presentation of the Research Plans. For the new applicant, it is very helpful to plan enough time for completion of the application so that it could be reviewed by others thoroughly knowledgeable in this particular area.

Once the application is submitted, the applicant should take advantage of opportunities to interact with the Executive Secretary of the Study Section to which the grant is assigned. Every institution's research office maintains a book identifying all of the Study Sections, the current members of the Study Section, and the Chairman. The assignment of the grant application to a particular study is an administrative one occurring at NIH upon receipt of the grant. It is to the applicant's advantage to attach a cover letter to the grant requesting, with specific justifications, a particular Study Section where his grant has the best chance for optimal review. Despite this effort, it is possible that the application may be assigned inappropriately. It is at the time of initial assignment that one can intervene in the system to ask for a different, more appropriate assignment. This cannot be done lightly and must be adequately justified in writing. It is also important to remember that the submission date of the application significantly precedes the actual review. Knowing the meeting dates of the Study Section may help the applicant provide an additional progress report or appropriate alterations in the experimental plan to the Executive Secretary prior to the actual meeting of the Study Section.

If the application is not successful, it is possible for the applicant to obtain this information and the critique very soon after the actual completion of the Study Section meeting. In rewriting the application, address the comments of the critique very carefully. It has been variously estimated that a rewritten grant carefully addressing the comments of the reviewers can improve a priority score by as much as 30 points. This is adequate incentive to work carefully and deligently to resubmit an application which did not quite make the "payline." Following the suggestions presented here should help to enhance the probability of success.

\section{ACKNOWLEDGMENTS}

I appreciate very much the helpful comments of the reviewers. This paper will be the basis for a presentation at the Association for Academic Surgery workshop on grant writing scheduled for the fall meeting.

\section{REFERENCES}

1. Cuca, J. M. NIH grant applications for clinical research: Reasons for poor ratings or disapproval. Clin. Res. 453-463, October 1983.

2. Henley, C. Peer review of research grant applications at the National Institutes of Health. 1. The assignment and referral processes. 2. Review by an initial review group. 3. Review by an advisory board/council. Federation of American Societies for Experimental Biology. Fed. Proc. 36: 2066-2068, 2186-2190, 23352338, 1977. 